\title{
Free-form Fresnel RXI-RR Köhler design for high-concentration photovoltaics with spectrum-splitting
}

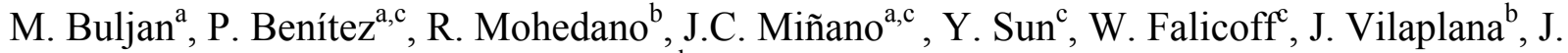 \\ Chaves $^{\mathrm{b}}$, G. Biot ${ }^{\mathrm{a}}$, J. López ${ }^{\mathrm{a}}$ \\ ${ }^{a}$ Universidad Politécnica de Madrid (UPM) CeDInt, Campus Montegancedo UPM 28223 Pozuelo, \\ Madrid, Spain \\ ${ }^{\mathrm{b}}$ LPI Europe, S.L., Edif. CeDInt Campus Montegancedo UPM 28223 Pozuelo, Madrid, Spain \\ ${ }^{\mathrm{c}}$ LPI-LLC, 2400 Lincoln Ave., Altadena, CA 91001
}

\begin{abstract}
Development of a novel HCPV nonimaging concentrator with high concentration ( $>500 \mathrm{x})$ and built-in spectrum splitting concept is presented. It uses the combination of a commercial concentration $\mathrm{GaInP} / \mathrm{GaInAs} / \mathrm{Ge} 3 \mathrm{~J}$ cell and a concentration Back-Point-Contact (BPC) silicon cell for efficient spectral utilization, and external confinement techniques for recovering the $3 \mathrm{~J}$ cell's reflection. The primary optical element (POE) is a flat Fresnel lens and the secondary optical element (SOE) is a free-form RXI-type concentrator with a band-pass filter embedded in it - Both the POE and SOE performing Köhler integration to produce light homogenization on the receiver. The band-pass filter transmits the IR photons in the 900-1200 nm band to the silicon cell. A design target of an "equivalent" cell efficiency $\sim 46 \%$ is predicted using commercial $39 \% 3 \mathrm{~J}$ and $26 \%$ Si cells. A projected CPV module efficiency of greater than $38 \%$ is achievable at a concentration level larger than $500 \mathrm{X}$ with a wide acceptance angle of $\pm 1^{\circ}$. A first proof-of concept receiver prototype has been manufactured using a simpler optical architecture (with a lower concentration, $\sim 100 \mathrm{x}$ and lower simulated added efficiency), and experimental measurements have shown up to $39.8 \% 4 \mathrm{~J}$ receiver efficiency using a $3 \mathrm{~J}$ cell with a peak efficiency of $36.9 \%$.
\end{abstract}

Keywords: Concentrator, spectrum splitting, high concentration, Köhler integration.

\section{INTRODUCTION}

At this time, the high concentration PV industry (HCPV) is focused on commercializing products based on triplejunction $(\mathrm{GaInP} / \mathrm{GaInAs} / \mathrm{Ge})$ solar cells, to take advantage of the high efficiency these cells provide. Increasing the cell efficiency is a clear path for reducing energy cost $(\mathrm{USD} / \mathrm{kWh})$ of concentrated photovoltaic energy $(C P V)$. Commercial cells from a variety of suppliers are close to $40 \%$ average efficiency, not far from the present world record $41.6 \%$ [1]. Two main sources of conversion losses are present in HCPV triple-junction cells: (a) they do not fully utilize parts of the solar spectrum above $900 \mathrm{~nm}$, and (b) there are reflection losses off the face of concentrator cells, mainly due to grid line shading. Even though competition between manufactures has resulted in a lowered triple-junction cell and assembly cost, these costs are still high to require high concentration $(>500)$ for the systems to be competitive in terrestrial applications.

Here we propose the development of a novel HCPV module with high concentration $(>500)$ and wide acceptance angle $\left(\alpha> \pm 1^{\circ}\right)$ that aims to reduce both sources of losses. The novel approach [18] uses the combined four junctions of a concentration (GaInP/GaInAs/Ge) cell and a Back-Point-Contact (BPC) concentration silicon cell for efficient spectral utilization, as well as an external confinement technique for recovering the reflected light from the cell. The silicon bandgap is nearly ideal for combining with the other three gaps (with a theoretical limit of $57 \%$ for two terminal devices (see [2])), which makes the combination very attractive. 
In four terminal operation of the ideal spectrum splitting combination of four junctions just mentioned, the current densities are very well matched (see Figure 1). In this configuration the silicon cell, whose full-spectrum current would be $44.4 \mathrm{~mA} / \mathrm{cm}^{2}$, is reduced to $27 \%$ of this level $(0.27=12.1 / 44.4)$, which implies that under equal geometrical concentration for both cells, the $3 \mathrm{~J}$ cell operates at its optimized 500x current gain, while the silicon cell runs at $0.27 \mathrm{x} 500$ or $135 \mathrm{x}$, close to its optimum [3].

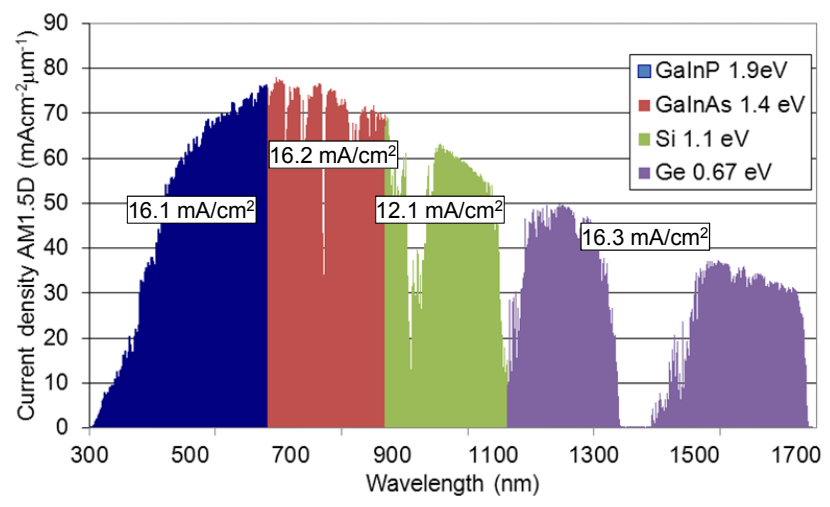

Figure 1 Band-gap calculation of maximum current densities (at 1-sun) of a four-terminal combination of a commercial concentration $\mathrm{GaInP} / \mathrm{GaInAs} / \mathrm{Ge} 3 \mathrm{~J}$ cell and a Back-Point-Contact (BPC) concentration silicon cell.

One of the most promising strategies for improving the utilization of the solar spectrum in multi-junction cells is the use of four or more p-n junctions. Different strategies have been followed to increase the number of junctions built into a solar cell such as: conventional epitaxial growth over a substrate (lattice matched and metamorphic), wafer bonding/ layer transfer process layer, and inverted metamorphic cells. The jury is still out on these approaches and other alternative ones. And it is still not known if the new devices can be built affordably and have the reliability and capacity to work efficiently under high concentration.

An alternative approach consists of using separate cells and dichroic optical filters. Compared to single cell solutions, this approach has the possibility of avoiding the current-mismatch losses through the use of multiple terminal configurations, and by eliminating the lattice matching prerequisites of monolithic growth. However, the development of a commercially vialable product based on this approach is very challenging due to probable increased material cost and system complexity. Even so, in the last few years, the dichroic beam-splitting approach has captured renewed interest $[4][5][6][7][8]$.

At present the External Quantum Efficiency (EQE) of cells is handicapped by the reflection losses from its face. These losses are very significant and severely limit the performance of the devices. In the case of $\mathrm{GaInP} / \mathrm{GaInAs} / \mathrm{Ge}$ solar cells, the front grid design is optimized to keep the series resistance joule losses low while minimizing the front metal grid shading factor. Reflection losses are produced by the reflection on the metallic front gridlines and the Fresnel reflection on the semiconductor surface. The front surface is not textured but is only AR coated. Further, the AR coating of present commercial cells is optimized to transmit in the 400-900nm range with reflection losses limited to 2-3\% (since the Ge junction has excess of current). However, the average reflection numbers over the full spectrum is in fact higher as a consequence of higher reflection values in the longer infrared region above $900 \mathrm{~nm}$.

Two different strategies have been used in the past to minimize the reflection losses. One consists in preventing the light from hitting the grid lines, for instance using refractive prismatic covers aligned over the grid lines (seminal work done by O'Neill, US Patent No. 4,711,972). There are various drawbacks to this approach (not recovering the light reflected on the semiconductor surface, needs very precise alignment, not effective under wide-angle that corresponds to high concentration), especially when a secondary optical element (SOE) is optically coupled to the cell as a secondary concentrator or homogenizer. Reflective covers have also been proposed, but their implementation also has practical challenges. A second strategy, referred to as External Confinement [9], is more compatible with high concentration. It 
consists of a mirror cavity that retroreflects the reflected light from the cell back to the cell. The cavities do not need precise alignment, but have the drawback that they still lose those photons absorbed on the grid lines.

The case of a BPC concentrator silicon cell is very different, since it has both contacts at the back of the cell surface and is not only AR coated, but randomly textured. As a consequence, the cell reflectivity is very close to zero at the wavelengths at which the silicon absorbs.

The need for competitive HCPV systems requires that the optical concentrator is designed not only to provide high concentration but also sufficient tolerances in order to keep the cost manufacturing low. A useful merit function for a $\mathrm{CPV}$ optic is the concentration-acceptance product [10], defined as:

$$
\text { (1) } C A P=\sqrt{C_{g}} \sin (\alpha)
$$

where, $\mathrm{Cg}$ is the geometric concentration and $\alpha$ the acceptance angle, often defined as the incidence angle at which the concentrator collects $90 \%$ of the on-axis power [11]. A more practical definition states it as the angle at which the generated photocurrent is $90 \%$ of the maximum (often achieved at normal incidence). This definition gathers all optical and electrical effects and is therefore more realistic. The acceptance angle $(\alpha)$ measures the total tolerance available between the system elements for a given $C_{g}$.

A novel HCPV nonimaging concentrator concept with high concentration $(>500 \mathrm{x})$ will now be presented. The system consists of a primary optical element (POE) that is a flat Fresnel lens, a secondary optical element (SOE) that consists of a monolithic free-form Köhler RXI-RR concentrator and an embedded cost-effective high-performance band-pass filter that reflects or refracts the light rays while crossing the device. In the RXI-RR SOE concentrator, the RR optic illuminates the BPC silicon cell, while the RXI optic illuminates the 3J cell. It is remarkable that the free-form RXI has achieved the highest reported CAP to date for refractive POE's [12]. Converting this advantage into the tolerance budget we are allowed to remove half of the system and exchange it with the external cavity in order to increase overall system efficiency, and still continue achieving the required high concentration level and acceptance angle.

The cell illumination in the new concentrator is asymmetric from one hemisphere, allowing the use of a confining cavity in the other hemisphere (as can be seen in Figure 5). This approach efficiently collects the light reflected by the grid lines and the semiconductor surface of the triple junction cell. The RXI-RR's free-form surfaces are calculated using an iterative process in three dimensions. The Köhler integration guarantees a uniform illumination of the two cells, free from spatial chromatic aberration.

\section{CONCENTRATOR DESIGN}

\subsection{Band-pass filter}

We have chosen for our spectrum splitting approach a design that utilizes two separate cells (four junctions in our case, three coming from a $3 \mathrm{~J}$ cell) and a dichroic optical filter. The band-pass filter (its working concept shown in Figure 2) is designed to be embedded in the secondary optical element RXI-RR. Its performance will provide the current balance between the bottom $(\mathrm{Ge})$ junctions, middle (GaInAs) junctions and top (GaInP), minimizing the losses not considered in the ideal calculation. The ideal calculation assumes pill-box type spectral splitting with idealized transmission and reflection zones with no absorption, which is near impossible to achieve in practice. In a real filter (schematically shown in red Figure 2b), the slopes of the edges of the transmission band depend on two parameters (see Figure 2a): the mean angle of incidence $\beta_{F}$, and the angular spread $\alpha_{F}$ of the light with respect to that mean direction. The slopes of the edges of the transmission band are progressively decreasing when the angular spread $\alpha_{F}$ increases, and the greater the mean angle of incidence $\beta_{F}$, the faster the degradation (for a given $\alpha_{F}$ ). 


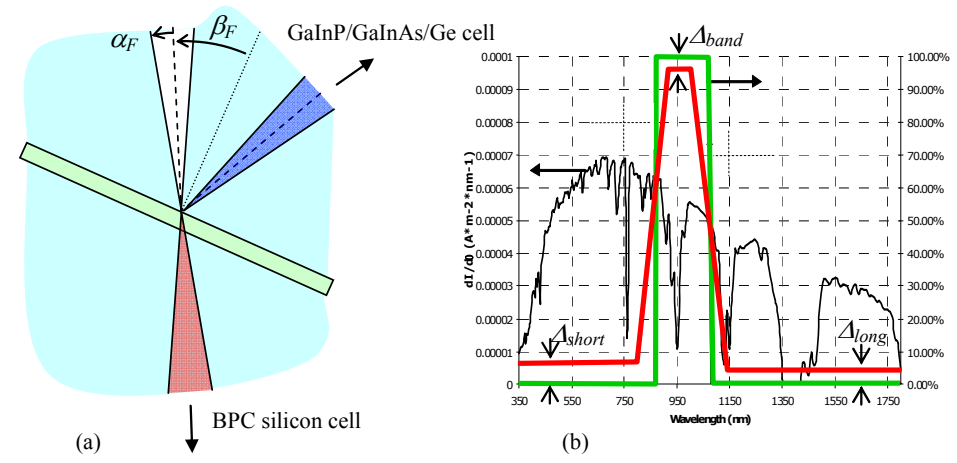

Figure 2 (a) Angle definitions on a schematic band-pass filter. (b) Spectral transmission of an ideal and a (schematic) real band-pass filter for an incident beam with non-null $\beta_{F}$ and $\alpha_{F}$ values.

The performance specification of filter embedded in RXI-RR SOE is limited by cost constraints. In order to achieve the price target we have restricted our concentrator design to operate using a flat filter. On one hand we would like the light to reach the filter with high concentration in order to lower the size and thereby cost (USD/m2) of the filter (that are meant to be deposited on large plane substrates), but on the other hand high concentration means wide incident angles $\alpha_{F}$, that smoothen the filter transmission curve, which compromises the current balance.

The necessity to moderate values of $\alpha_{F}$ suggests limiting the concentration on the filter to the minimum that is cost effective ( 40-50x). That is why an SOE is utilized after the filtering, which provides an additional 10-12x concentration on the cells. We have selected $\beta_{F}>25^{\circ}$ and $\alpha_{F}>5^{\circ}$, when the surrounding media is a dielectric material $(n \approx 1.5)$.

The EQE of the four junctions and its aggregation curve for the four-terminal case are shown in Figure 3a. The dip in the aggregation curve near the silicon band-gap corresponds to photons which are sent to the silicon cell but not efficiently absorbed by it. The effectiveness of the external confinement cavity is clear in Figure $3 b$ by the comparison of this curve with the original EQE's of the triple-junction cell.

(a)

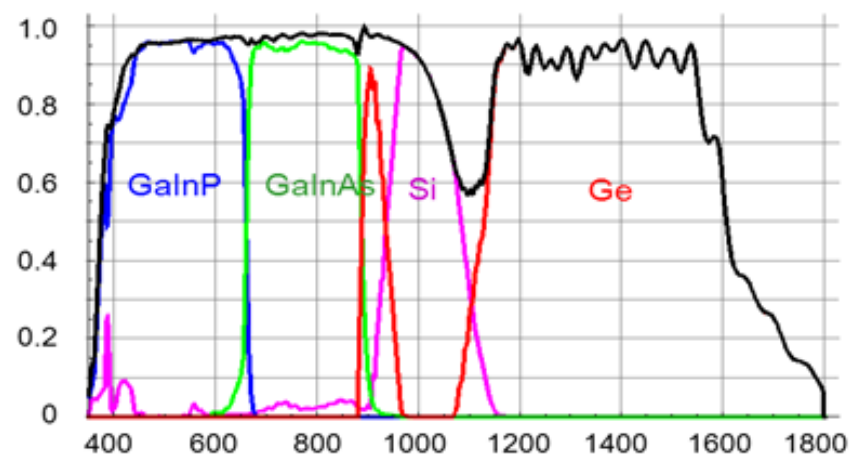

(b)

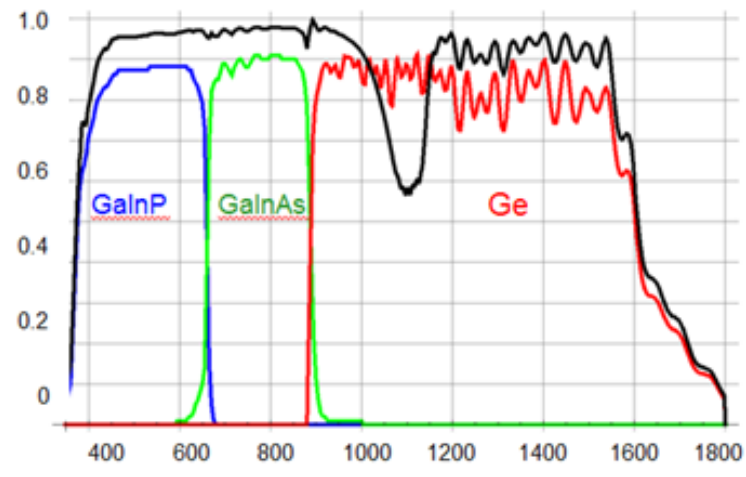

Figure 3 (a) EQE of the four junctions of an RXI-RR design for four terminals, and its aggregation. Our modeling indicates that this design should achieve about $46 \%$ efficiency under AM1.5d with $39 \% 3 \mathrm{~J}$ cell and $26 \%$ silicon cell. (b) The same aggregation overlapped with the original EQE of the triple-junction cell where the increase of the EQE due to the external confining cavity can be appreciated.

\subsection{Confining cavity}

Apart from possibility to increase efficiency by improving solar spectrum utilization through the use of dichroic filter spectrum splitting, the new approach adds an external confining cavity to recover the lost reflected light from the $3 \mathrm{~J}$ cell. 
As already mentioned, this feature consists of a a mirrored cavity that collects the light reflected by the cell either by the gridlines or by the semiconductor surface and sends it back to the cell. (Light absorbed on the grid lines is not recovered.)

For present triple-junction cells, the light reflected on the semiconductor surface is specular (the cell surface roughness is very small, similar to good optical mirrors), while the light reflected on the grid lines has a significant diffuse component caused by the grid line geometry and roughness. However, the roughness and imperfections of the grid line surface still keep the same cylindrical symmetry, as a good approximation (see Figure 4). Therefore, the direction of the scattered light conserves the vector component along the grid line, and a light ray is scattered inside the surface of a reasonably narrow cone. This is important for the design of the concentrator and the external cavity (which traditionally has assumed random Lambertian scattering). As was stated before in this approach the illumination of the cell must be done from one hemisphere, while the cavity occupies the other (asymmetric cavity).
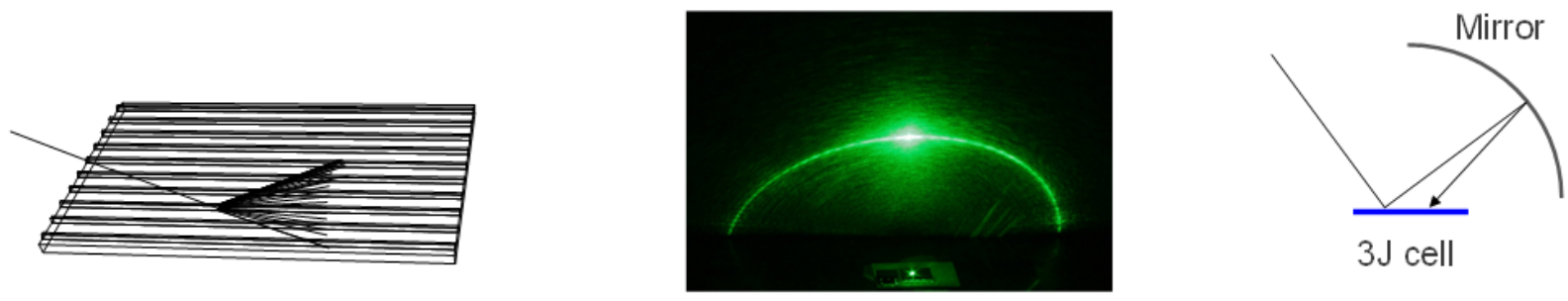

Figure 4 The irregularities and roughness of the gridlines of a commercial concentration triple-junction cell has approximately cylindrical symmetry, which allows efficient external confinement with an asymmetric cavity.

In case of our design we are investing a half of the system to enhance the overall efficiency by means of external confining cavity. Having sufficient tolerance budget in our optical design, we are in a position to do it as will be explained in greater detail in next subsection.

\subsection{Fresnel-RXI Köhler concentrator}

The concept of the Fresnel RXI-RR Köhler concentrator is shown on Figure 5. The primary optical element (POE) is a flat Fresnel-Köhler lens, and the secondary optical element (SOE), a monolithic free-form Köhler RXI-RR concentrator, comprising an embedded band-pass and an external confining cavity. The letters naming the device originate from the usual nomenclature of the SMS method [13], and refer to the type of surface $(\mathrm{R}=$ refraction, $\mathrm{X}=$ reflection, $\mathrm{I}=$ Total Internal Reflection) that reflects or refracts the light rays while crossing the device. RXI-RR free-form surfaces are calculated with the SMS method using an iterative process in three dimensions.

In the RXI-RR SOE concentrator, the RR illuminates the BPC silicon cell, while the RXI illuminates the 3J cell (see Figure 5). The triple junction cell is asymmetrically illuminated from one hemisphere, allowing the use of a confining cavity in the other hemisphere which efficiently collects the light reflected by the grid lines and the semiconductor surface of the triple junction cell. 

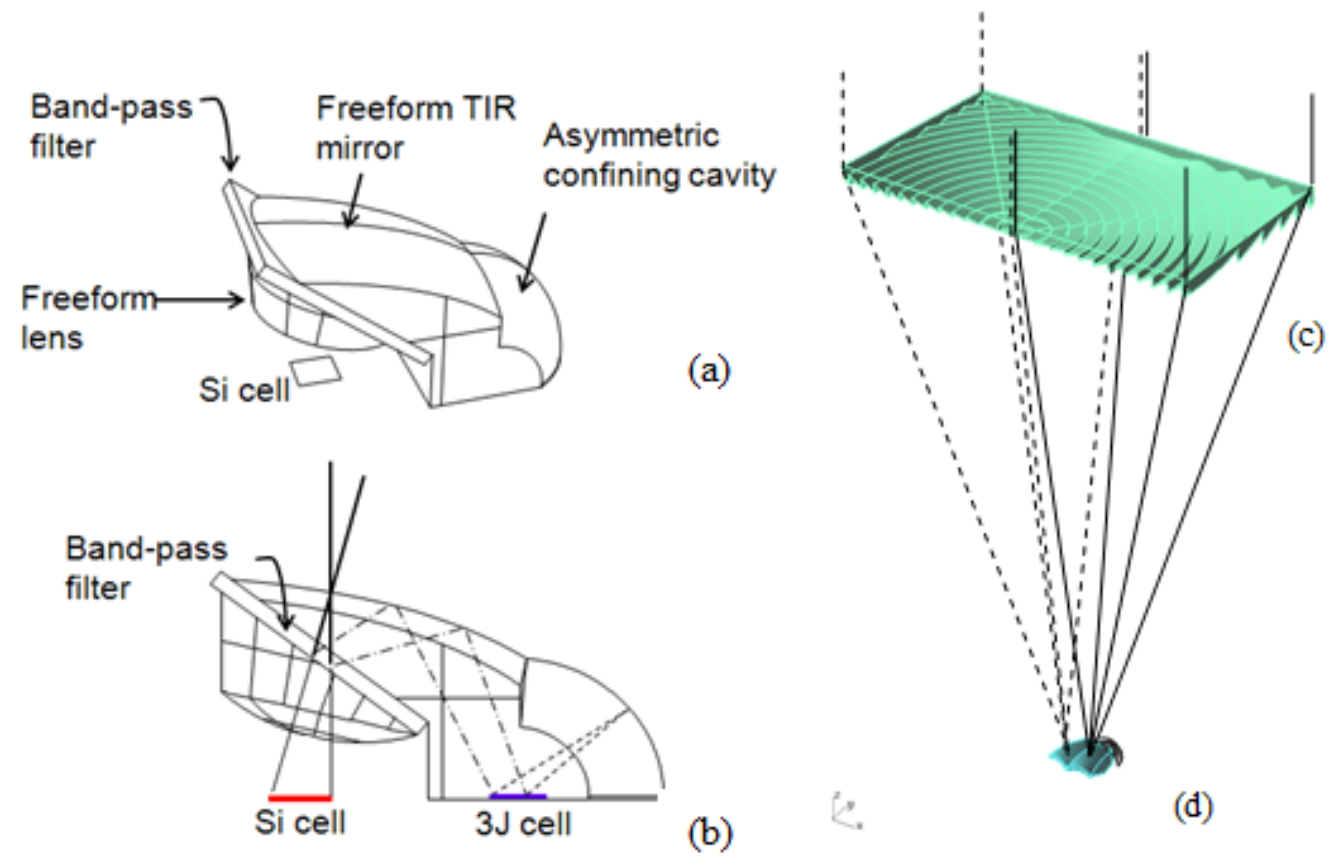

Figure 5 (a) \& (b) 1-fold Köhler RXI-RR SOE concentrator, including the flat band-pass filter and the GaInP/GaInAs/Ge and BPC silicon cells. (c) Asymmetric 2-fold Köhler Fresnel lens illuminating the corresponding 2-fold RXI-RR SOE (d).

The free-form RXI was originally developed for Solid State Lighting in the automotive industry [16], and it has been recently designed for HCPV applications [12]. It is very important to underline once again that the free-form 4-fold RXI has the highest "CAP budget" among concentrators with a refractive POE (Figure 6) and we are using this benefit to increase the system overall efficiency.


Figure 6 Ray tracing on the SOE of a 4-fold Fresnel-RXI Köhler (b) The Fresnel-RXI Köhler concentrator surpasses the Concentration Acceptance angle product (CAP) of all the existing commercial concentrators using a flat Fresnel lens as a POE (extracted from [12]).

The Fresnel-Köhler families for HCPV (named FK, XRK, XXRK) have been introduced by LPI in recent years [14][15], as novel high-performance concentrators that provide not only high concentration and high acceptance angles (i.e., high CAP), but which also exhibit excellent uniformity of illumination on the square cell without the effects of chromatic aberration on the cell. In the design of Köhler concentrators, we select the number $N$ Köhler channels, which indicate the number of paired sectors in the POE and SOE. The design is then referred to as an $N$-fold design, and $N$ usually ranges between 1 and 4 . In this project we will consider the 1-fold and 2-fold options. 
The design presented here is based on the previously developed free-form 4-fold design RXI recently introduced as a Köhler secondary for HCPV [12]. The Köhler integration technique guarantees in this design, as well as in other previously presented ones, uniform illumination on both cells (see Figure 7), free from spatial chromatic aberration.

There are various advantages of the RXI-RR design over prior beam splitting systems such as a reduction in complexity. First, the filter, the SOE for the BPC silicon cell (i.e., the RR) and the SOE for the triple-junction cell (i.e., the RXI) form a single piece of dielectric, which simplifies its mounting. Secondly, the two cells are located on the same plane, simplifying the heat management and wiring. Thirdly, there are no optical surfaces in contact with the cell rim, which differs from other traditional HCPV secondary optics (such as prism homogenizers). This makes the encapsulation of the cells much easier and more robust, since there is no threat of light loss due to meniscus effects by the silicone rubber coupler, making it more suitable for high-yield in mass production [15].

There are alternative SOE's architectures that have been considered. Freeform RXI-RI' ${ }^{2}$ configuration allows the Silicon and $3 \mathrm{~J}$ cell to be located not only coplanar but also closer than in the RXI-RR, so they can share the receiver substrate. This design, with a geometrical concentration of $625 \mathrm{x}$ for the $3 \mathrm{~J}$ cell and 560x for the Si cell, has a modeled optical efficiency of $85 \%$ for both the MJ and silicon cells (no AR coating considered). In addition it exhibits a well-balanced acceptance angle of $\pm 0.9^{\circ}$ for both cells as well as achieving uniform irradiance distribution on $3 \mathrm{~J}$ and Si cells as evident in Figure 7.
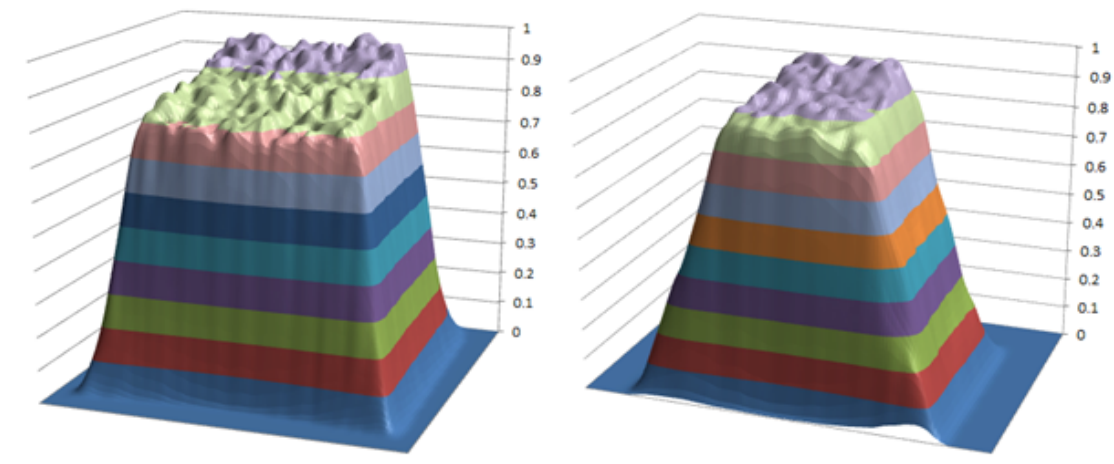

Figure 7 Irradiance distributions on the 3J and Si cells (a.u.), respectively.

\section{PROOF-OF-CONCEPT PROTOTYPE}

A proof-of-concept prototype that is based on a simpler optical concept has been manufactured by LPI and UPMCeDInt. The prototype (seen in Figure 8) is comprised of an RXI prism, with a 41-layer stop-band dichroic filter (deposited on the long right angle prism leg proximate to the $3 \mathrm{~J}$ cell), a GaInP/GaInAs/Ge cell and a BPC concentration silicon cell. The entry aperture (hypotenuse of the prism) is AR coated with a proprietary design by LPI. The short side of the right triangle leg of the prism also has the same AR coating, which insure high transmission of the prism to the silicon cell, which has an air gap between itself and the prism. The filter was designed to reflect the light whose wavelengths range between $900-1150 \mathrm{~nm}$ to the silicon cell, transmitting the light whose wavelengths are outside this range to the $3 \mathrm{~J}$ cell. This prototype doesn't include a confining cavity, so reflected radiation of the semiconductor and grid-lines is sent back through the filter to the silicon cell.

As there is no confining cavity in this prototype, reflected radiation from the semiconductor and grid-lines is sent back through the filter to the silicon cell. This lowers the expected efficiency due to two facts: 1) the loss of the 3J-reflected photons over $1150 \mathrm{~nm}$ (used by the Ge junction in the original RXI-RR and RXI-RI ${ }^{2}$ design), 2) the light recycled towards Si cell below $900 \mathrm{~nm}$ is not as profitable as if it was recycled back to the MJ cell, as the voltage of the Si cell is 
lower than that of the top and middle subcells. Although, this prototype still illustrates the high potential of the spectrum splitting concept.

Optical transmission measurements of the manufactured filter coating have shown important deviations from the design values. From measured values we could predict that there is a higher reflection of photons below $900 \mathrm{~nm}$ to the silicon cell. The measurement of the manufactured AR coating showed that it produces a transmission $\sim 96 \%$ over $900 \mathrm{~nm}$, instead of the designed $98 \%$.
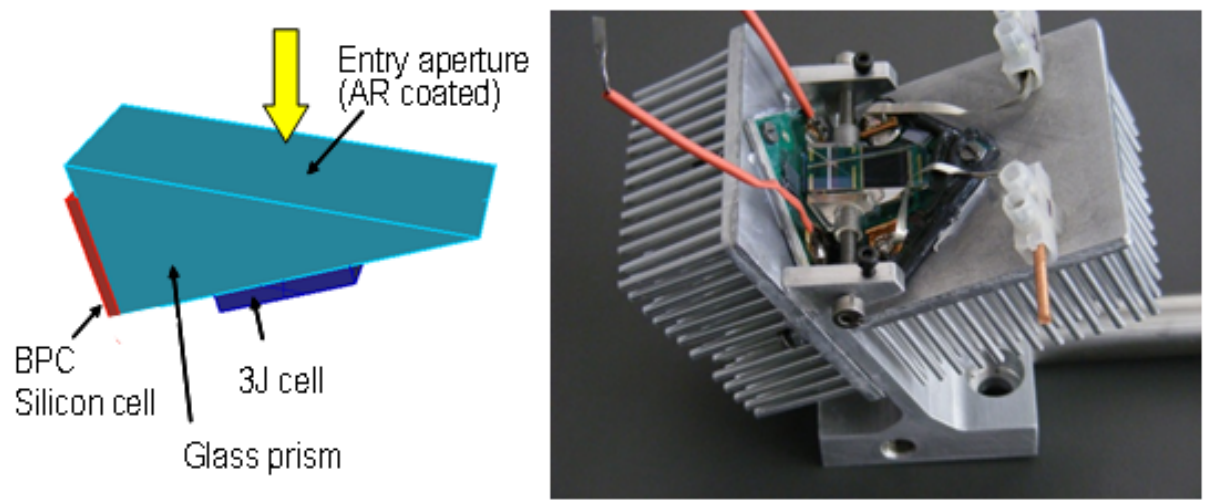

Figure 8 Proof-of concept prototype 4J module.

Two 3J cells were selected and electrical measurements were carried out in conjunction with a standard flash tester at 50 $\mathrm{W} / \mathrm{cm}^{2}$ and AM1.5d spectrum. They showed efficiencies of $39.4 \%$ and $39.5 \%$. One of them was then attached to the RXI prism inside the proof-of-concept prototype using a silicone adhesive. (The BPC silicon cell was also installed in the prototype.) Afterwards measurements were taken under the real sun on March $21^{\text {st }}$ in Madrid, using a concentrator test rig that was developed to produce a well-defined square irradiance distribution of $\sim 6.5 \times 6.5 \mathrm{~mm}^{2}$ with $7.33 \mathrm{~W}( \pm 2 \%)$ at @ $900 \mathrm{~W} / \mathrm{m}^{2}$, to be fully inscribed in the receivers to measure, producing $\sim 10 \mathrm{~W} / \mathrm{cm}^{2}$ average concentration on the $3 \mathrm{~J}$ cell area. Under that concentrator test rig, the reference $3 \mathrm{~J}$ cell alone showed a peak $36.8 \%$ efficiency ( $36.9 \%$ corrected for Tcell $=25^{\circ} \mathrm{C}$ ),

Measurements performed under the same concentrator rig with 4J RXI prism receiver assembly gave 39.8\% (see Table 1) with $4 \mathrm{~T}\left(39.7 \%\right.$ corrected for Tcell $\left.=25^{\circ} \mathrm{C}\right)$, and $39.7 \%$ with $2 \mathrm{~T}$. Thus, output peak-power gain of the $4 \mathrm{~J}$ over the $3 \mathrm{~J}$ was about $8 \%$. Both four terminal and two terminal cases are studied, taking into account higher efficiency and less spectral sensitivity in one case or cost savings in other case, respectively. 


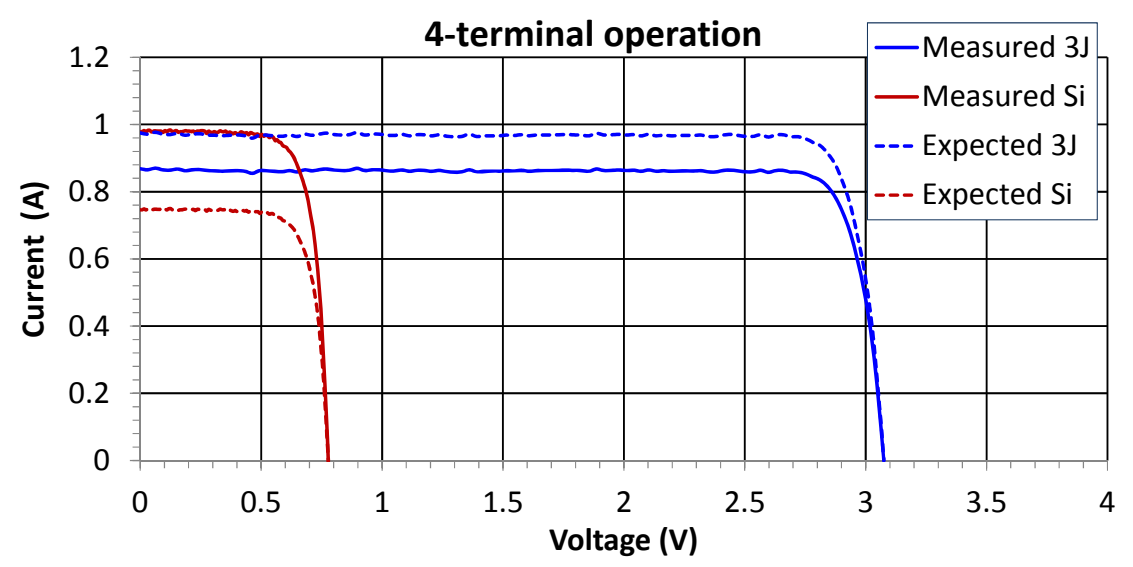

Figure 9 Peak-efficiency I-V curves (measured and expected) of the $4 \mathrm{~J}$ receiver module.

\begin{tabular}{lccc}
\hline DATE & \multicolumn{3}{c}{$3 / 21 / 2011$} \\
\cline { 1 - 3 } Time & \multicolumn{2}{c}{$12: 15$} & \\
\cline { 1 - 3 } CELL & 3J & Si & \\
Isotypes Jratio top $/ \mathrm{mid}$ & 0.95 & & \\
Isc $\left(@ 900 \mathrm{~W} / \mathrm{m}^{2}\right)$ & 0.87 & 0.98 & \multirow{2}{*}{ Total: } \\
Voc & 3.07 & 0.78 & $39.8 \%$ \\
FF & 0.88 & 0.75 & \\
\hline Efficiency & $32.0 \%$ & $7.8 \%$ & \\
\hline
\end{tabular}

Table 1 Measured peak efficiency of the $4 \mathrm{~J}$ receiver module.

The results we have until now are very significant and promising, especially taking into account the problems found in the coatings manufactured in this first iteration. The unexpected higher current values measured on the silicon cell compared with the expected values (see Figure 9) need further research. Overall we are encouraged by the results and are confident that the target of a $13 \%$ gain will be achievable in the next iteration once the design of the filter is properly implemented (see Table 2). In the next phase we plan to use a concentrator rig with higher concentrations $(\sim 300 \mathrm{x})$. Commercial 3J cell and BPC Si cells performance followed by higher efficiencies is closer to the optimum one they are designed for in this new configuration of concentrator rig.

\section{CONCLUSIONS}

The main objective of this work is to increase the overall system efficiency and reliability of CPV system through the use of solar spectrum division thereby reducing lifetime energy cost (USD/kWh). Our approach was guided by an analysis of the causes of receiver/optics losses and resulting improvements in the optical system design through more efficient use of the available energy in the bottom cell of a $3 \mathrm{~J}$ cell.

We have presented a novel high-concentration Köhler SOE concept with an integrated dichroic flat filter for coplanar commercial 3J cell and BPC Si cells, with expected equivalent cell efficiency of $46 \%$ from 39\% 3J cells ( $+17 \%$ gain). 
The first simple prototype showed an $+8 \%$ efficiency gain, and the next iteration (with an improved filter) targets a $+13 \%$ gain and $43 \%$ efficiency at 300x. Future efficiencies of over $50 \%$ could be attainable with custom designed $3 \mathrm{~J}$ (with better Ge sub-cells) and Si cells (with better IR response).

The next steps are focused on building a demonstrator able to attain a $13 \%$ efficiency boost (see Table 2 ). The additional efficiency gain is based on increasing the concentration to 300x at the receiver entry, so that the cells work more efficiently. The higher concentration brings additional challenges, such as dealing with the assembly and alignment of parts. Amonix is supplying champion cells and is sharing the costs for the new hardware needed.

\begin{tabular}{lcc}
\hline & Measured & Expected \\
\hline Reference 3J & $36.9 \%$ & $37.4 \%$ \\
4J prism & $39.7 \%$ & $42.5 \%$ \\
\hline Gain & $\mathbf{+ 8 \%}$ & $\mathbf{+ 1 3 \%}$ \\
\hline
\end{tabular}

Table 2 Measured efficiencies of the $3 \mathrm{~J}$ reference cell and $4 \mathrm{~J}$ prism receiver in $4 \mathrm{~T}$ operation (corrected for $\mathrm{Tcells}=25^{\circ} \mathrm{C}$ ).

\section{AKNOWLEDGMENTS}

The authors thank V. Garboushian, A. Plesniak and G. Kinsey, from Amonix Inc. for their support for this work. The authors also thank G. Sala, R. Herrero, R. Nuñez and J. Leloux, from IES-UPM, for their help to characterize the 3J cells outdoors and indoors.

Support for this work partly comes from the EC, Spanish Ministry MITYC and C.A.M. under projects SSL4EU (Grant agreement $n{ }^{\circ} 257550$, FP7/2007-2013), V-SS and SMS-IMAGING (TSI-020100-2011-445 and 372, Plan Avanza 2011) and 4LL (PIE/468/2010), respectively. 


\section{REFERENCES}

[1] M.A. Green, et al. "Solar cell efficiency tables". Prog. Photovolt: Res. Appl.; 17(5): 320-326 (2009).

[2] R.R. King et al. "Progress in High-Efficiency III-V Multijunction Concentrator Solar Cells", DOE Solar Program Review Meeting, Denver, April 16-19, (2007).

[3] A. Slade et al., "Issues encountered in the manufacture of $125 \mathrm{~mm}$ thick, $26 \%$ efficient silicon solar cells in microelectronics foundries", 19th EU PVSEC, (2004).

[4] A. Barnett et al., "Very High Efficiency Solar Cell Modules", Prog. Photovolt: Res. Appl.; 17:7583(2009).

[5] M. A. Green, A. Ho-Baillie. "Forty three per cent composite split-spectrum concentrator solar cell efficiency", Prog. Photovolt: Res. Appl.; 18:42-47 (2010).

[6] L.M. Fraas, et al. "Toward 40\% and Higher Multijunction Cells in a New Cassegrain PV Module", 31st IEEE PVSC, Florida, USA (Jan. 2005).

[7] E. Shifman, US pat. application 2008/0000516.

[8] B. Mitchell et al. "Four-junction spectral beam-splitting photovoltaic receiver with high optical efficiency" Prog. Photovolt: Res. Appl., Online, july, (2010).

[9] J. C. Miñano, "Optical confinement in photovoltaics,“ in Physical Limitations to the Photovoltaic Solar Energy Conversion, A. Luque and G. L. Araújo, eds. (Hilger, Bristol, UK,1990).

[10] Benitez P., J.C. Miñano, "Concentrator Optics for the next generation photovoltaics". Chap. 13 of A. Marti \& A. Luque. [Next Generation Photovoltaics: High Efficiency through Full Spectrum Utilization], Taylor \& Francis, CRC Press, London, (2004).

[11] Luque A., [Solar Cells and Optics for Photovoltaic Concentration], Adam Hilger, Bristol, (1989).

[12] M. Buljan et al., "Improving performances of Fresnel CPV system: Fresnel-RXI Köhler concentrator", 25th EU PVSEC, Valencia (2010).

[13] R. Winston, J.C. Miñano, P. Benítez, "Noinimaging Optics” (Academic Press-Elsevier, 2004).

[14] P. Zamora, et al, "Advanced PV Concentrators”, 34th IEEE PVSC, Philadelphia, (2009).

[15] P. Benitez et al. "High performance Fresnel-based photovoltaic concentrator," Opt. Express 18, (2010).

[16] J.C. Miñano et al. "Free-form integrator array optics". Nonimaging Optics and Efficient Illumination Systems II, SPIE. R. Winston and J, Koshel Ed., Vol. 5942-12. San Diego, USA, (2005).

[17] US and International Patents Pending including: P, Benítez et al., "Photovoltaic Device" PCT publication WO 2010/016930 February 2, (2010).

[18] International patent pending. 